\title{
EL DESARROLLO DE LA EUGENESIA EN CUBA
}

\author{
Armando García González \\ Instituto de Historia - C.S.I.C. Duque de Medinaceli, 6 - 28014 Madrid (España)
}

\section{RESUMEN}

En este artículo se estudian las temáticas más generales que caracterizaron a la eugenesia cubana, como su incorporación a la enseñanza, los problemas racial e inmigratorio y las campañas higiénico-sanitarias, así como las posiciones más significativas adoptadas por algunos de sus relevantes defensores.

\section{SUMMARY}

In this paper are studied the essential problems that the characterized Cuban eugenics, like its corporation to education and teaching, the radical and inmigratory problems and the hygienicsanitary campaigns, as well as the most significant positions adopted by some of their most important defenders.

El movimiento eugénico en Cuba tuvo relevancia sin precedentes en relación con otros países americanos, si exceptuamos los Estados Unidos de América, posiblemente a causa de sus vínculos estrechos con esta nación (que incluía dependencia económica y política), pero también debido al desarrollo económico alcanzado por la Isla en las primeras décadas del siglo XX; desarrollo que si bien no era comparable con su poderoso vecino del Norte y diversos países europeos, se destacaba entre algunos del área panamericana, mucho más atrasados. Algo que podía aplicarse asimismo al nivel logrado en Cuba en cuanto a las ciencias médicas, ya significativas desde el siglo XIX. Como es lógico, tal movimiento se vio condicionado a los distintos fenómenos sociales, políticos y económicos que caracterizan las cuatro primeras décadas del siglo $\mathrm{XX}$, tanto internos como externos; pudiendo señalarse entre ellas las intervenciones militares en Cuba, sus gobiernos políticos, crisis económicas, el exterminio de los Independientes de Color, la Constitución de 1940, etc.; a los que se unieron sucesos como las dos guerras mundiales, la guerra civil española, y otros muchos que no es posible explicitar en esta apretada síntesis.

La eugenesia cubana no está en modo alguno divorciada de la que se desarrollaba en el mundo entero, si bien parece tener más vínculos, por razones obvias, con los Estados Unidos, al menos hasta la caída del gobierno de Gerardo Machado en 1933, 
aunque también los eugenistas caribeños conocieron y se relacionaron con sus homólogos en México, Perú, Argentina y Venezuela, por sólo citar algunos casos. No obstante, a partir de 1936 y hasta 1958, la nueva hornada de eugenistas cubanos mostrará posiciones más cercanas a la izquierda, con aparente independencia de los norteamericanos o por lo menos sin una dirección tan evidente y directa como la que se observa en el primer grupo, sin que esto quiera decir que no tuviesen, en algunos casos, como ellos, ideas extremas en cuanto a ciertos presupuestos eugénicos.

Sin entrar en muchos detalles, que en cierto modo han sido ya tratados en investigaciones anteriores ${ }^{1}$, hay que decir que la eugenesia cubana, como la internacional, se ocupó de variados temas que abarcaban aspectos tales como la inmigración, la higiene y salud pública, cuestiones legales, educación y enseñanza, sociología, literatura, etc. En ese sentido se generaron, debatieron y publicaron diversos trabajos y se participó en los principales congresos nacionales e internacionales que se produjeron en el área panamericana, donde se abordó la eugenesia, ya como tema principal, ya colateral. Al parecer Cuba tuvo su representante en la Federación Internacional de Eugenesia, desde 1912, conjuntamente con otras naciones que ingresaron en esa fecha, como fueron Argentina, Bélgica, Dinamarca, Italia, Francia, Noruega y Estados Unidos ${ }^{2}$. Eugenistas cubanos participaron en las Conferencias Panamericanas de Eugenesia y Homicultura, celebradas en La Habana y Buenos Aires, en 1927 y 1934, respectivamente, y también en los Congresos Internacionales de Eugenesia efectuados en 1921 y 1932 en New York, pero también estuvieron presentes en cónclaves que tocaron de algún modo el asunto de la eugenesia, como fueron los congresos de inmigración e emigración, los de higiene, protección de la infancia, etc. Fueron además los fundadores de la homicultura, «ciencia» creada por Domingo Ramos y Eusebio Hernández a partir de la puericultura divulgada por Adolfo Pinard, que, como la eugenesia, pretendía el mejoramiento de la población desde la infancia hasta el estado adulto, aunque a veces por senderos muy variados.

Los primeros trabajos de eugenesia en Cuba comienzan a aparecer en la Isla inmediatamente después de efectuado el Primer Congreso Internacional de 1912, si bien ya se conocían las ideas de Galton desde 1877, enseñándose en la Universidad en la década del ochenta, para luego ser reintroducidas al menos desde 1904 en asuntos de puericultura. Pero es, desde luego, a partir de 1913 cuando la eugenesia recibe un fuerte impulso en Cuba, con la memoria de A. F. Tredgold «El estudio de

1 Cfr. NARANJo ORovio, C. y García GonZÁLeZ, A. (1996) Medicina y racismo en Cuba: la ciencia ante la inmigración canaria. Tenerife, Taller de Historia; GARCíA GONZÁLEZ, A. y ÁlvAREZ PELÁEZ, R. (1999) En busca de la raza perfecta: eugenesia e higiene en Cuba: 1898-1958. Madrid, CSIC; así como la bibliografía utilizada y mencionada por estos autores.

2 LAUGHLIN, H. H. (1934) «Historical background of the Third International Congress». En A Decade of progress in Eugenics Scientific Papers of the Third International Congress of Eugenics. Baltimore, The Williams \& Wilkins Company. 
la eugénica $»^{3}$, Nicolás Amador (1914), Adolfo Pinard (1914), Eugène Apert (1916) y otras varias presentadas por los cubanos Nicolás Gómez de Rosas y Trinidad Sáinz de Llavería en el Primer Congreso Médico Nacional, efectuado en La Habana en 1914, y más específicamente la difusión dada por el médico Domingo Ramos en congresos y conferencias, por el oftalmólogo Juan Santos Fernández en diversas revistas científicas, y por el antropólogo y médico Arístides Mestre en la enseñanza universitaria, todos por esos años. Son abundantes en las segunda y tercera décadas y primeros años de la cuarta y luego, de forma más esporádica, entre 1942 y 1958 . La introducción de las ideas eugénicas en Cuba se produjo más o menos simultáneamente, tanto en relación con los temas médicos, como con los biológicos (también por 1914) y los sociológicos en la enseñanza universitaria (a partir de la tercera década), pero también por abogados que trataron las cuestiones legales, y por antropólogos que abordaban la criminalidad, la prostitución y otros males que aquejaban a la sociedad cubana, en consonancia con los temas analizados en otras partes del mundo. Trataremos de agrupar los aspectos más generales que abordó la eugenesia cubana a fin de realizar algunas reflexiones generales.

\section{EUGENESIA, HOMICULTURA E HIGIENE}

Como fue palmario para algunos países, la atingencia entre la eugenesia y la higiene devino de hecho algo natural, si se tiene en cuenta las pretensiones de mejorar la población (entendida muchas veces como raza, clase social, etc.), sobre todo en los países americanos que adoptaron una postura más cercana a la que Raquel Álvarez ha denominado y nosotros hemos asumido como «latina» (más preocupada por las condiciones ambientales) que a la «ortodoxa», derivada de las opiniones galtonianas más puras, que insistía mayormente en los factores hereditarios. Si bien algunos eugenistas cubanos, como Ramos, hicieron hincapié en la importancia de la herencia como aspecto fundamental para erradicar los males que provocaban la «degeneración» de la población, tanto él, como la mayoría de los eugenistas cubanos concedieron gran relevancia a las cuestiones higiénico sanitarias para lograr ese objetivo.

El grupo encabezado por Ramos, incluyó variadas figuras con objetivos a veces distantes; tal es el caso, por ejemplo, del mencionado Hernández, médico obstetra y general de la segunda guerra independentista cubana, de quien Ramos había sido discípulo, como ambos lo fueron a su vez de Adolfo Pinard. Las ideas de Eusebio Hernández, aunque apoyadas en campañas higiénico-sanitarias, más profundas en su concepción quizá que las de Ramos, descansaban en el hecho de que Cuba debía

\footnotetext{
3 TREDGold, A. F. (1913) «El estudio de la eugénica», (tr. de The Quarterly Review por Ramón de Armas Colón), Cuba Contemporánea, La Habana (enero), t. 1, nº 1, pp. 191-218.
} 
pasar por cambios estructurales políticos. El propio Hernández estuvo por cierto tiempo involucrado en la política antes de lograr las pretensiones eugenistas de mejorar su población. Hernández no sólo había luchado por la independencia de Cuba, sino que era consciente del estado de pobreza y analfabetismo de la población cubana y asumió una actitud digna ante la corrupción gubernamental y la politiquería de «generales y doctores», que caracterizaron esas décadas en Cuba. Sus intentos, conjuntamente con Ramos, de fundar desde 1910 un Palacio de la Homicultura, que aglutinase todas las disciplinas médicas, higienistas y eugénicas, no halló eco en los gobiernos de turno de los cuales demandó determinadas sumas de dinero; si bien es cierto que se llegaron a crear varios hospitales con esa orientación, como fue por ejemplo el Hospital de Matanzas, pero de muy reducido resultado en relación con las pretensiones de aquél.

El resto de los eugenistas encabezados por Ramos comprenden ideas más comunes a éste: entre ellos estaban Francisco María Fernández, Juan Santos Fernández, Enrique Núñez, Matías Duque, Enrique Varona Suárez, José Antonio López del Valle, Antonio Barrera Condom y otros, quienes desplegaron intensas campañas a favor de presupuestos eugenistas e higiénicos, como fueron el certificado médicoprenupcial, la esterilización de los criminales y débiles mentales, el control de los inmigrantes, los concursos de higiene, entre otros. A este grupo se debieron importantes logros en relación con la higiene, como fueron la creación de un Negociado de Higiene y otro de Homicultura, en el Ministerio de Sanidad y Beneficencia (luego de Salubridad y Asistencia Social) y campañas que tuvieron además carácter benéfico y político, como fueron los Concursos de Maternidad y Fertilidad Eugénica, la creación de dispensarios, creches y Gotas de leche, en resumen de varias instituciones para la atención del niño, la mujer embarazada, etc. Ellos favorecieron y organizaron también la Primera Conferencia Panamericana de Eugenesia y Homicultura, celebrada en La Habana, en 1927, de la cual surgió una Oficina que radicó en el aludido ministerio y en la Academia de Ciencias Médicas, Físicas y Naturales de la Habana. Su director, hasta 1933 en que partió al exilio, fue el también presidente de la Academia, Francisco María Fernández. Si bien la Oficina parece haber tenido poca actividad, al menos organizó y apoyó la Segunda Conferencia Panamericana, de Buenos Aires, en 1934. Tanto en estos, como en otros cónclaves de esos años, fue Domingo Ramos el máximo representante de Cuba en relación con estos temas, divulgando de paso la homicultura como genuina creación cubana.

Este primer grupo de eugenistas cubanos concebía la higiene como un triángulo isósceles, cuya base estaba constituida por la herencia y la eugenesia, siendo los lados del triángulo la sanidad (medio ambiente) por un lado y la homicultura (desarrollo individual) por el otro. La maneras de actuar ${ }^{4}$ y la acción social de la homicultura

4 Cfr. figuras reproducidas al final de este artículo. 
estaba dirigida a la madre en cuanto a los cuidados prenatales (matrifeticultura) y preeducacionales (matrinaticultura); al maestro, los cuidados educacionales (puericultura), y al club los preconcepcionales (patrimatricultura), pregenitales (pregenitocultura) y postgenitales (postgenitocultura). Por otra parte, la interrelación higienebiología-homicultura se establecía de la siguiente manera:

\section{ESTUDIO BIOLÓGICO DE LA HOMICULTURA}

Higiene individual (Homicultura)

\author{
Pregenital \\ (Pregenitocultura)
}
Genital Preconcepcional (Patrimatricultura) Postconcepcional

\section{Prenatal \\ (Matrifeticultura)}

Postanatal Preeducacional

(Matrinaticultura)

Educacional

(Puericultura)
Ser vivo

(susceptible de cultivo)

Mamífero placentado

Mamífero placentado

(simbiosis o parasitismo filio materno)
Sexuado

(con dos células necesarias para la reproducción)

\section{Hombre}

(con cerebro susceptible de educación completa $)^{5}$

\section{Pluricelular}

(células divididas en germinales y somáticas)

Es curioso cómo la homicultura fue rápidamente aceptada por los países panamericanos, así como el Código de Eugenesia y Homicultura creado por Ramos y sus seguidores, aunque este tuvo importantes críticas, sobre todo en lo concerniente al control de los inmigrantes, a la aplicación obligatoria del certificado médicoprenupcial y a la esterilización. Ramos pretendía determinar la entrada de inmigrantes a los países latinoamericanos mediante el análisis de los gametos de éste para intentar hallar en él las características «cacogénicas» que le invalidaban, o las eugénicas favorables que le favorecían a la hora de ser aceptado por los países receptores.

5 Esquema presentado al VI Congreso Médico Latino Americano y expuesto en la Exposición Internacional de Higiene del mismo y ante la Comisión de Higiene de la V Conferencia Panamericana. RAMOS, D. F. (1924) Cuba en la higiene internacional y finlaísmo. Habana, Imp. La Propagandista. 


\section{ARMANDO GARCÍA GONZÁLEZ}

Ideas que estaban más acordes con algunos eugenistas norteamericanos y con la política de selección aplicada en ese país, pero en desacuerdo con las opiniones de otros eugenistas latinoamericanos que vieron en éstas y otras medidas una forma de discriminación y abuso de poder, como lo dejó claro el médico higienista peruano Enrique Paz Soldán.

\section{EL CONTROL DE LA INMIGRACIÓN}

La eugenesia fue para el ámbito latinoamericano de inmediata aplicación a las cuestiones y preocupaciones inmigratorias. Las grandes campañas que se desarrollaban en los países del área para aumentar la población ante las reales o no demandas de mano de obra para trabajar en la agricultura, la industria, etc. Los intereses de ciertas clases de obtener mano de obra barata se hicieron eco de las opiniones de Alberdi y de otros pensadores sobre la relación del desarrollo de una nación por el aumento de la población. Si bien muchos eugenistas pensaban que más que del número, tal desarrollo dependía de la calidad de la población que se seleccionase para recibir como inmigrante. Esta inmigración preocupaba desde varios puntos de vista: por un lado por las enfermedades, taras y malformaciones que introducían en los países receptores, que luego eran transmitidas ya directamente por contagio, ya mediante la herencia, cuando contraían matrimonios con los «naturales» o teniendo hijos de sus familias cuando venían con ellas. De ese modo se exageró en ocasiones las enfermedades introducidas por los inmigrantes y en otros casos se minimizó y hasta se violaron las más elementales reglas de control sanitario, como sucedió en Cuba, durante la segunda y tercera década del siglo XX con la entrada de jamaicanos y haitianos, a veces por partes de la costa de Cuba, donde no existía ese control. Otras veces sólo se hacía un análisis superficial, o la pretendida cuarentena en Triscornia se abreviaba, debido a las gestiones de los grandes terratenientes y dueños de ingenios, principales promotores de la introducción de mano de obra barata para desempeñar las labores agrícolas.

Ello provocó a veces la protesta de instituciones como ocurrió, por ejemplo, en 1924, en Cuba con la Academia de Ciencias por las enfermedades que agravaban la ya difícil situación de la población cubana, en especial, la campesina.

\section{EL PROBLEMA RACIAL}

Para las clases medias que no tenían interés directo en la entrada de mano de obra barata, la preocupación central se encaminó no sólo hacia la adecuada selección del inmigrante, teniendo en cuenta razones médicas, sino también hacia otras de índole 
social, política y económica. Por ello, en los primeros años del siglo XX Cuba insiste en mejorar sus condiciones de vida y trabajo para que los inmigrantes se asienten en el país, integrándose a la población y permaneciendo de ese modo el capital en la Isla. Esto es posible observarlo, por ejemplo, en las Conferencias de Beneficencia y Corrección de la Isla de Cuba, efectuadas desde 1906 hasta 1911. Las políticas llevadas a cabo por los gobernantes que satisfacían los intereses de terratenientes y dueños de ingenios norteamericanos y cubanos de importar jornaleros para trabajar en la agricultura cañera, sobre todo a partir de la segunda década, pero también la situación de crisis económica cubana, su dependencia del monocultivo de la caña de azúcar y otros factores políticos internos, dieron lugar al incremento de conflictos raciales, como el exterminio de los Independientes de color en 1912, masacrándose miles de negros y mulatos en Cuba. La entrada de jamaicanos y haitianos en la Isla significó para diversos médicos, higienistas, eugenistas e intelectuales cubanos una forma de acrecentar los graves conflictos existentes en el país y por tanto la propia concepción de la nacionalidad cubana. Concepción que en algunos casos se retrotrajo a las valoraciones de José Antonio Saco, Domingo del Monte, Francisco de Arango y Parreño y otros pensadores cubanos del siglo XIX que concebían más la nacionalidad ceñida a la raza blanca. De ahí que, como aquellos, intentasen blanquear la población, proponiendo la entrada de población blanca a la Isla, ya fuesen españoles (peninsulares y canarios) $\mathrm{u}$ otros europeos. Un ejemplo de ello se observa en algunas memorias del oftalmólogo cubano Juan Santos Fernández ${ }^{6}$.

Fue también una forma de enfrentar los intereses de los grandes terratenientes, como es posible observar en algunos de los representantes de la clase media cubana (médicos, historiadores, etc.), al proponer la inmigración por familias, de cultura y lengua similar a la cubana, a la que se debían entregar tierras, que llegasen a ser con el tiempo de su propiedad; ideas que, como es lógico, no estaban acordes con los intereses de los grandes terratenientes, aludidos anteriormente. En algunos casos, representantes de esa clase media se pronunciaron contra la política de los Estados Unidos que favorecía tal situación en Cuba. Así, algunos eugenistas fueron bastante claros al condenar la política norteamericana en Cuba, como fue por ejemplo el homeópata y literato cubano Juan Antiga, quien en su intervención en la Primera Conferencia Panamericana en La Habana en 1927, criticó la doctrina Monroe pidiendo una nueva política más justa, racional, tolerante y humana para los pueblos latinoamericanos. Razón por la cual -suponemos nosotros- no se incluyera su intervención en las Actas de esa conferencia publicadas al año siguiente ${ }^{7}$.

Del mismo modo para algunos países americanos, con poblaciones indígenas importantes la inmigración constituía también una forma de blanquear la población. Es

6 Naranjo Orovio, C. y García GonZález, A., Opus cit.

7 Cfr. AnTIGA, J. (1931) Escritos políticos y sociales, Madrid, Taller Espasa-Calpe, S. A., pp. 153-161. 


\section{ARMANDO GARCÍA GONZÁLEZ}

una época donde aún imperan ideas procedentes del XVIII y XIX sobre la degeneración del criollo, del mestizo y del mulato, creyéndoseles incapaces de gobernarse a sí mismos, como pensaban Montesquieu, Gustave Le Bon y otros autores. La América mestiza y mulata era también, para algunos intelectuales latinoamericanos, degenerada e infantil, incapaz de mantener instituciones políticas que la representasen, necesitando ser gobernada por caudillos, como pensaban Francisco García Calderón, Laureano Valenilla Lanz, José Gil Fortuol, entre otros, y en Cuba Alberto Lamar Schweyer ${ }^{8}$, o en otros casos requiriendo la higiene racial propugnada por los nazis, como defendió el médico peruano Enrique Gamio en las Jornadas Peruanas de Eugenesia celebradas en ese país en 1939 y 1943. Posiciones extremas a las que se opusieron algunos eugenistas cubanos, de tendencia izquierdista, como el sociólogo Roberto Agramonte y el obstetra José Chelala-Aguilera9 ${ }^{9}$

\section{EUGENESIA Y MEDICINA}

Como ya hemos visto, los médicos eugenistas se interesaron por los asuntos concernientes a la higiene, la sanidad y la inmigración por razones obvias. Como la eugenesia pretendía la selección de los mejores para evitar la transmisión hereditaria de taras y enfermedades, el tema de la herencia se convirtió en relación con estas cuestiones de particular interés para los galenos. La creencia de una heredodisposición para ciertas enfermedades como la tuberculosis u otros males como la sífilis, el alcoholismo, etc., perduró en muchos médicos durante gran parte de la primera mitad del siglo XX. Aunque pronto se supo la diferenciación entre enfermedades congénitas y verdaderamente hereditarias, el término supracitado tardó en ser abolido. Entre otras razones, porque de algunas enfermedades se desconocía su verdadera naturaleza 0 tenía diversos orígenes, como era el caso de la epilepsia, pero también porque convenía a los eugenistas destacar el peso de la herencia en muchos de estos trastornos para lograr sus objetivos de control social, o la obtención de reformas por parte de los gobiernos para mejorar las población. De hecho, muchas veces se exageraban considerablemente el número de los tarados y «degenerados» en las distintas naciones, y lo mismo sucedía con la criminalidad, la prostitución, la vagancia y otros males,

8 Cfr. GARCÍA CALDERÓn, F. Les Democraties latines de l'Amerique.; TRIANA, R. Democracia y tropicalismo, GIL FORTUOL, J. Filosofía Constitucional; ARRAGAY, L. La anarquía argentina y el caudillismo; RoJAS, L, M. La culpa de Henry Wilson en el desastre de México; EDWARDS, A. La fronda aristocrática. En Cuba, LAMAR SCHWEYER, A. (1927) La biología de la democracia. La Habana, Editorial Minerva. En contra de esas opiniones, véase AGRAMONTE, R. (1927), La biología contra la democracia. La Habana, Imprenta La Milagrosa. Para un análisis de estas cuestiones, véase GARCíA GONZÁLEZ, A., Los grilletes de la ciencia: Discriminación y antirracismo en la enseñanza de la ciencia en Cuba (inédito).

9 García GonZÁleZ, A. y Álvarez PeláeZ, R. Opus cit. 
cuyas causas se debían en realidad a factores sociales, económicos y políticos, pero no hereditarios. Así y todo algunos eugenistas cubanos se hicieron eco de los estudios de Dugdale y otros autores, quienes concebían la heredabilidad de estos males, poniendo como ejemplos los famosos casos de las familias Kallikak y Jukes. Así como asumieron también las de la herencia del genio y del talento propugnadas por Galton y sus seguidores.

Si bien para la posición ortodoxa la herencia fue, en ese sentido, factor primordial sobre la acción del medio ambiente, cuando convenía aludir a éste en relación con la enseñanza de la eugenesia, la higiene, etc. entonces era tenida en cuenta. Para los países latinoamericanos, no era posible separar ambos factores si se piensa en las masas campesinas y obreras pobres, la gran tasa de mortalidad infantil, el alto grado de analfabetismo, así como las abismales diferencias sociales, políticas, económicas y culturales de sus poblaciones, etnias y clases.

Por otra parte, los conocimientos acerca de la herencia no parecen haber sido comprendidos en toda su magnitud en las primeras décadas que siguieron al redescubrimiento de las leyes de Mendel en 1900 por Tschermak, Correns y De Vries. En Cuba, las primeras referencias a esas leyes datan, al parecer de 1909, aplicadas a cuestiones sociales por el doctor Diego Tamayo; si bien se discuten más en detalle por vez primera tres años después en la Academia de Ciencias Médicas, Físicas y Naturales de la Habana, por el oftalmólogo suizo Anton Lutz, entonces residente en Cuba. De inmediato se aplicaron a diversas disciplinas médicas, así como al campo de la biología, de la pedagogía, de la antropología, etc. Y, por supuesto, se interconectaron con la eugenesia, máxima aspiración a que podían llegar los estudios sobre la herencia en humanos, como antaño se había logrado en los trabajos efectuados sobre plantas y animales.

También algunos antropólogos abordaron la eugenesia en relación con la criminalidad, por las taras hereditarias que creían ver en éstos y en las prostitutas, como fue por ejemplo, el cubano Israel Castellanos, a quien se deben muchos trabajos sobre estas temáticas, apoyándose no sólo en Lombroso, sino también en las ideas de Magnan en cuanto a los estigmas de degeneración que creían ver en los criminales. Taras degenerativas que se aplicaron asimismo a los enfermos mentales, como revelan algunos trabajos del médico alienista y biólogo Arístides Mestre.

En algunas ocasiones el estudio de la herencia sirvió para combatir las teorías racistas que reverdecían en la década del treinta con la política racial implantada por los nazis a partir de 1934. En ese sentido puede destacarse, por ejemplo, el trabajo de Octavio Montoro «Nuevas orientaciones en los estudios sobre la herencia y en el concepto de razas» ${ }^{10}$, leído dos años después en la Academia de Ciencias de La Ha-

10 MONTORO, O. (1936) «Nuevas orientaciones en los estudios sobre la herencia y en el concepto de razas». Anales de la Academia de Ciencias Médicas, Físicas y Naturales de la Habana, t. 37, pp. 35-56. 


\section{ARMANDO GARCÍA GONZÁLEZ}

bana. Momento en que también en Cuba se recrudecen las opiniones racistas no sólo contra negros y mulatos cubanos, sino también en apoyo a grupos falangistas y fascistas, llegándose a formar, incluso, en la Isla un partido nazi en 1938, más tarde disfrazado de Quinta Columna y rápidamente diluído por el general Fulgencio Batista, entonces en la presidencia de Cuba.

\section{EUGENESIA Y LA ENSEÑANZA DE LA BIOLOGÍA}

La eugenesia se apoyó en las concepciones biológicas de su tiempo, haciendo un buen uso de ellas. De Darwin, por ejemplo, se asumieron sus conceptos sobre la selección natural y la lucha por la existencia, pero no su teoría de la pangénesis, desacreditada por su primo Francis Galton. En cambio la teoría sobre el plasma germinal desarrollada por Weismann, sí fue ampliamente utilizada. Lo mismo sucedió posteriormente con las leyes de Mendel, y las concepciones sobre el apoyo mutuo de Pedro Kropotkin. Todas estas concepciones de la eugenesia internacional están presentes también en la cubana, que, por lo común, sólo se mencionan o explican muy someramente.

Herencia, eugenesia y biología fueron conjugados a partir de 1914 por el médico alienista y antropólogo cubano Arístides Mestre, al entrar en contacto con las obras de De Vries, Morgan y otros biólogos que se ocuparon de la herencia. Mestre introdujo en seguida estos conceptos en la enseñanza universitaria, en la cátedra de antropología que desempeñaba interinamente desde 1910 hasta 1922, cuando pasó a ocuparla oficialmente. Sus obras de texto sobre antropología y biología de esos años reflejan las fuentes consultadas así como las explicaciones que en torno a esas materias se impartían por él. Su labor en ese sentido fue continuada por la bióloga Mercedes Mayo, quien produjo trabajos donde se refería a la eugenesia y a su papel en la mejora de la población, así como el también biólogo cubano Julio Fernández de la Arena, quien impartió cursos y conferencias en la década del cincuenta sobre herencia y eugenesia, proponiendo la creación de una oficina que se encargase de esos estudios en Cuba.

En la enseñanza secundaria (institutos de segunda enseñanza) otros biólogos y profesores elaboraron asimismo obras de texto que recogían aspectos acerca de la herencia y la eugenesia, algunos de ellos desde una orientación religiosa, como fueron, por ejemplo, los libros de los jesuitas Pelegrín Franganillo y Faustino García. Otros, en cambio, asumían una actitud distante y no comprometida desde el punto de vista ideológico o ante los debates éticos que se producían ante temas como la esterilización, el aborto y el control de la natalidad, que criticaron aquellos biólogos religiosos, al tiempo que distinguían, desde ese punto de vista, una eugenesia positiva de otra negativa. 


\section{EUGENESIA Y LA ENSEÑANZA DE LA SOCIOLOGÍA}

También se impartió la eugenesia en relación con la sociología en la universidad de la Habana y en los institutos de segunda enseñanza, posiblemente a partir de 1926; aunque se recogen en las obras de texto desde 1939. Correspondió a Roberto Agramonte esa labor en las dos décadas siguientes. Mientras que en la enseñanza secundaria otros profesores como Gustavo Amigó Jansen (en institutos religiosos), Francisco Domenech, José Nodarse y varios más, siguiendo estos últimos las mismas tendencias de la obra de Agramonte que servía de libro base, desplegaron su labor a partir de los primeros años de la década del cuarenta. Agramonte, Domenech y otros sociólogos adoptaron asimismo las ideas hereditarias de su época (Mendel, De Vries, Morgan), así como las de eugenesia, con tendencia izquierdista e incluso socialista, como es el caso de Domenech. Se proyectaron en contra de las corrientes racistas y fascistas de la época, a través de las ideas que reflejaron en esas obras de texto, pero también de los trabajos que orientaban y pedían a los alumnos. Así, aunque no sabemos hasta qué punto la enseñanza de la eugenesia influyó en los educandos, ni qué consecuencias tuvo, sí creemos que la lucha desplegada en contra de las opiniones racistas y fascistas fue un aspecto positivo para las concepciones de los estudiantes y por tanto de la conciencia nacional cubana de entonces. También ignoramos hasta qué punto pudo influir en otras partes de Latinoamérica, si se tiene en cuenta que la sociología de Agramonte (en dos volúmenes) fue empleada además en la enseñanza en otras naciones, como México, etc.

\section{LA EUGENESIA Y LOS DEBATES ÉTICOS}

Algunas medidas defendidas por los eugenistas como la esterilización, la experimentación en criminales, el certificado y examen médico prenupcial, el aborto y el control de la natalidad, y en general los temas relacionados con la sexología, tuvieron carácter polémico, por cuanto atentaban o rozaban cuestiones éticas. Curiosamente, a la eugenesia se le recuerda y vincula más con estas cuestiones que con la más abundante (al menos para América Latina) masa de argumentaciones que abarcó referente a los problemas higiénicos, sanitarios, sociales. Posiblemente ello se deba al excesivo uso que hicieron los nazis de algunas de esas medidas. Pero se olvida, o se desconoce que quienes comenzaron, por ejemplo, la esterilización de criminales y débiles mentales fueron los norteamericanos, desde la temprana fecha de 1907. En 1948 ya se calculaba para ese país más de cuarenta mil esterilizaciones ${ }^{11}$. No se tiene en cuenta

11 «Esterilización de dementes y débiles mentales en los E.U.», Vida Nueva, La Habana, 1950, Año XXIV, $2^{\circ}$ época, t. LXV, $\mathrm{n}^{\circ} 1$, p. V. Véase también «leyes de esterilización», Ibidem, 1938, Año XII, $2^{\circ}$ época, t. XLI, ${ }^{\circ} 1$, pp. I-II.; «Eugenética y estrilización obligatoria», Medicina de Hoy, La Habana, 1936, Año I, n ${ }^{\circ}$, p. 415 . 
tampoco, por lo regular, que tales medidas se pusieron en práctica también en otros países. Noticias recientes reflejan que en algunas naciones europeas se aplicó durante décadas la esterilización. En Suecia, por ejemplo, se esterilizaron a la fuerza personas no sólo deficientes mentales, sino hasta algunas que tenían trastornos leves como miopía, e incluso por la ley aprobada en esa nación en los años veinte con el fin de «mejorar la raza», aquellas que tenían rasgos «agitanados». Método que continuó hasta tiempos recientes. Entre 1935 y 1976 se efectuaron esterilizaciones forzosas con el fin de eliminar tipos raciales que se consideraban inferiores; Suiza y Noruega realizaron prácticas similares y lo mismo, al parecer, continuó haciendo Austria, sometiendo a esterilización forzosa a deficientes mentales y a otras personas consideradas «asociales» ${ }^{12}$. Investigaciones actuales revelan igualmente que en Francia se aplicaron estas medidas en unas quince mil mujeres, a quienes se efectuaron ligaduras de trompas en contra de su voluntad: «Un cociente intelectual inferior a 80, un carácter considerado 'agresivo', el ser sorda y muda, el haber sido violada por el padre, el tener malas calificaciones escolares y, en definitiva, el vivir en un medio familiar desintegrado y de gran pobreza material son algunos de los motivos por los que se ha esterilizado a jóvenes en esas instituciones especiales ${ }^{13}$ ».

Todo ello no quita, desde luego, que el nacionalsocialismo le confiriese a estas prácticas, sin embargo, el grado de genocidio.

Que sepamos ni en Cuba ni en España se llegaron a realizar esas prácticas de forma obligatoria, si bien en el primer país contó con algunos defensores, como los aludidos Francisco M. Fernández, Juan Santos Fernández, Octavio Mañalich, Matías Duque y otros. En cuanto a la experimentación en humanos (condenados a muerte), se produjo en la prensa en Cuba un debate, a favor y en contra, de aplicárseles el cáncer. Según cuentan, el propio Duque, -cuyo padre había muerto a causa de esta enfermedad- se aplicó células cancerígenas en la piel, pues no creía en la inducción de las células enfermas en las sanas. A nivel de otros medios de comunicación, como la radio, se produjeron asimismo debates en torno a la aplicación del certificado prenupcial y de uniones matrimoniales entre enfermos de lepra, el control de la natalidad y la reglamentación de la prostitución, analizados en la Junta de Sanidad y hasta en la Cámara de Representantes. Todas estas cuestiones quedaron sin embargo reducidas a cenáculos científicos y políticos, pero no trascendieron -en lo que sabemos- a la mayor parte de la población. Algo parecido sucedió con otros temas eugénicos, como los abortos, y especialmente con los denominados criminales o clandestinos.

Los abortos constituyeron -y constituyen hoy- un asunto controvertido. No todos los eugenistas se proyectaron del mismo modo en cuanto a los mismos. Algunos se manifestaron antiabortistas (médicos, abogados y biólogos religiosos o no), otros

12 Cfr. «Unas 15.000 mujeres deficientes han sido esterilizadas en Francia sin su consentimiento». El País, Madrid, 11 de septiembre de 1997, p. 20.

13 Ibidem. 
estuvieron a favor de los mismos (abortistas) y otros los aceptaron para ciertos casos como violaciones, personas enfermas de tuberculosis y epilepsia, entre otros. En Cuba todas estas posiciones son observables, en consonancia con otras partes del mundo en diversos galenos, profesionales e intelectuales. Si bien la mayoría de los eugenistas cubanos parecen aceptar esta última variante (Ramos y otros), los intelectuales religiosos, se inclinaron, por su parte, a favor de la primera variante. Estos últimos también se manifestaron en contra del control de la natalidad, que propugnaban eugenistas como el ginecólogo Chelala-Aguilera y Mario Villegas, calificando a los métodos anticonceptivos como prácticas neomalthusianas; del mismo modo que ocurría en otras partes del mundo. A pesar de la posición de la iglesia católica, que tenía cierta ascendencia en Cuba (de hecho se condenaron a varias mujeres por abortos clandestinos, sobre todo de las clases más pobres) se aplicaron esos medios anticonceptivos (Chelala refiere que él intervino en más de cuatrocientos casos) y se efectuaron muchos abortos clandestinos por médicos que no fueron nunca procesados.

\section{LA EUGENESIA Y LA REALIDAD SOCIAL CUBANA}

El grupo encabezado por Chelala-Aguilera, perteneciente al Partido Ortodoxo, de tendencia izquierdista, conjuntamente con el famoso político cubano Eduardo Chibás, quien terminó suicidándose de un balazo durante una alocución radial, y otros denunciaron las condiciones precarias en que vivían la población obrera y campesina de Cuba: la falta de vivienda, la pobreza, ínfimas condiciones higiénicas, bajos salarios (pueden verse al respecto sus artículos de fines de la década del treinta y principios de la siguiente en la revista Bohemia), pero también de otras de mayor alcance como las drogadicciones, los efectos de la segunda guerra mundial y otras.

Sin embargo, la mayor parte de las campañas eugenistas e higiénico-sanitarias no podían alcanzar a los sectores a quienes más debían afectar: la alta mortalidad infantil cubana, analfabetismo y situación precaria de sus condiciones de vida y trabajo, ya señaladas, demandaban cambios más profundos desde el punto de vista social, político y económico. Las luchas obreras de esa época por sus reivindicaciones fueron en definitiva más fructíferas.

\section{¿SE REVITALIZA LA EUGENESIA ACTUALMENTE?}

La amplia gama de criterios generados por la eugenesia y más exactamente la riqueza de detalles que presenta en muchos de sus defensores, por lo regular pertenecientes a las clases medias y altas, reflejan que no es posible simplificar los presupuestos eugénicos más conocidos (que por lo regular coinciden con medidas extre- 
mas o negativas de algunos de sus representantes), toda la amplia gama de criterios que la caracterizó. Del mismo modo, no es posible aglutinar tampoco dentro de la misma bolsa la eugenesia promovida en naciones desarrolladas, como las europeas, conjuntamente con la llevada a cabo en países de América Latina, cuyas realidades sociales eran muy distintas: alto índice de analfabetismo y mortalidad infantil, grandes masas de obreros campesinos e indígenas pobres y discriminados, etc. Así, parte de la eugenesia no pudo ignorar estas realidades, y más de uno fue consciente de la importancia de realizar grandes cambios políticos, económicos y sociales si se quería en verdad mejorar la población, como pretendían los eugenistas.

Eso no quita para que las políticas (y políticos) nacionalistas se aprovecharan de algunos de sus presupuestos extremos (en el cual no había consenso ni muchos menos entre los eugenistas, como no la había tampoco entre médicos, biólogos, abogados, sociólogos) a fin de lograr, con mezquinos intereses egoístas y de poder, control social, político y económico de distintos países. No quita tampoco para que fuese y sea válido el cuestionamiento sobre los asuntos éticos que trajo y trae consigo la aplicación de prácticas y políticas científicas por personas, instituciones y gobiernos poco recomendables.

Los debates más recientes en torno al genoma humano y a la clonación han traído de nuevo a la luz algunos de los presupuestos eugénicos vinculados con las posiciones extremas, sobre todo en relación con la experimentación humana. Sin embargo, la realidad es que desde los tiempos de auge de la eugenesia (y aun antes) ha existido una continuidad en cuanto a las preocupaciones por el uso que se hace (y se pretende hacer) de la manipulación científica. Los fines no siempre justifican los medios. Así no creemos que, en aras de un supuesto mejoramiento, haya que recurrir al crimen, que supone la utilización y manipulación de embriones humanos o de conflictos psicológicos, morales y emocionales que pueda generar, aunque para justificar dicha aplicación se recurra a toda clase de eufemismos. Desgraciadamente las necesidades individuales, pero también las ansias de gloria, de poder y de riquezas, son motores impulsores de la actividad incontrolada de ciertas personas en el terreno científico y, lo que es tan grave como esto, que la actividad y logros científicos generados con miras altruistas sean convertidos por esa clase de personas en escalones para alcanzar aquellos y no estos objetivos. 


\section{CONCEPTO INTEGRAL DE LA HIGIENE}

(Eugenesia - Sanidad - Homicultura)

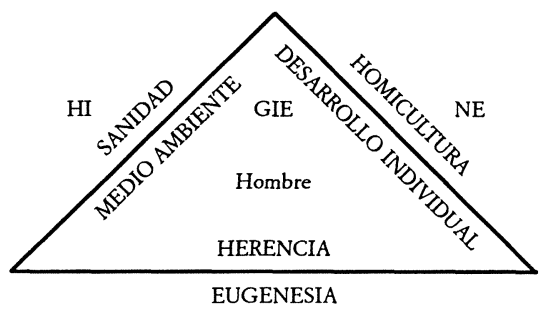

Complemento de un esquema de Conklin sobre constitución biológica del hombre presentado al VI Congreso Médico Latino Americano y reparto en la Exposición Internacional de Higiene de la V Conferencia.

RAMOS, D. (1924) Cuba en la bigiene internacional y el finlaísmo, Habana, Imprenta La Propagandista

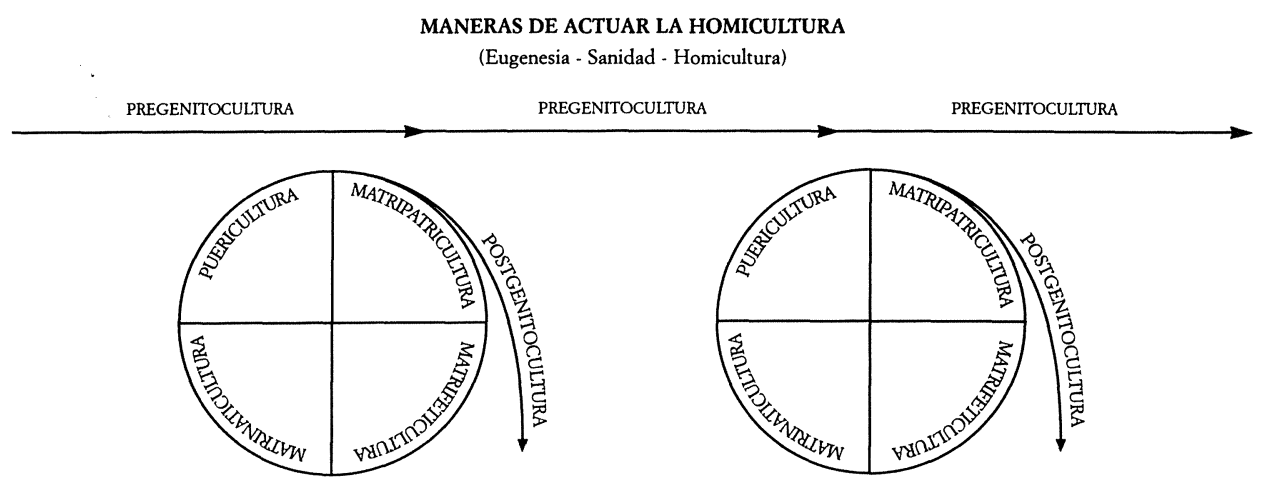

Presentado al II Congreso Internacional de Eugenesia, al IV Congreso Médico Latino Americano y expuesto ante la Exposición Internacional de Higiene del mismo y la Comisión de Higiene de la V Conferencia Panamericana.

RAMOS, D. (1924) Cuba en la bigiene internacional y el finlaismo, Habana, Imprenta La Propagandista 


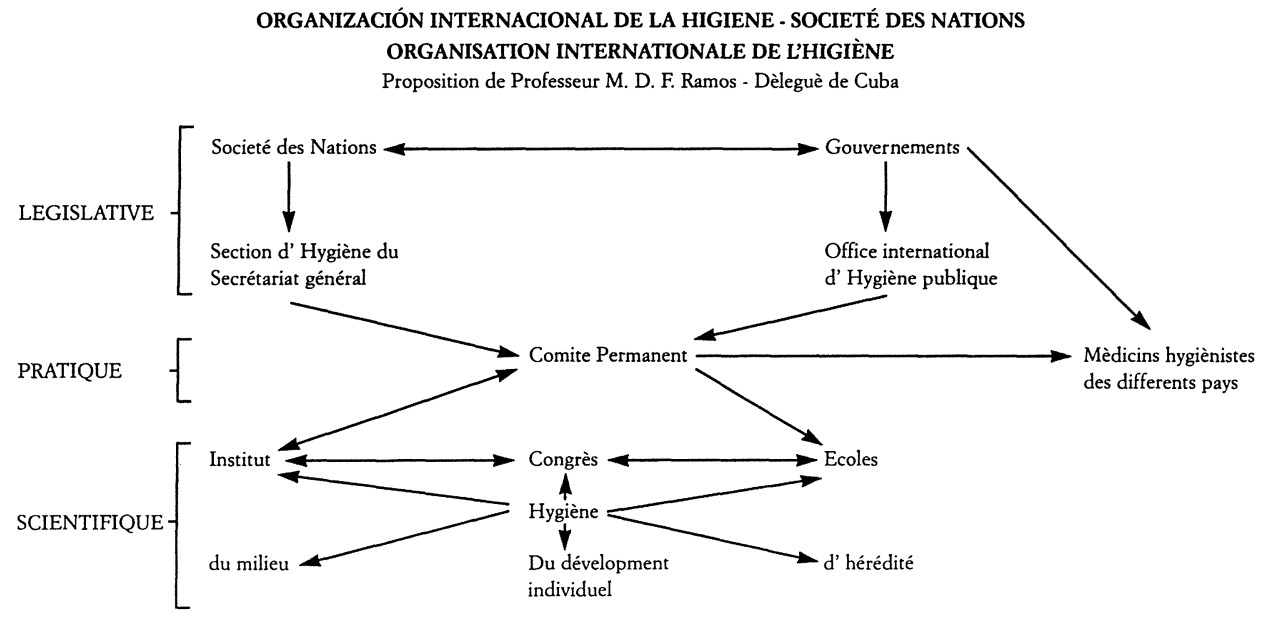

Presentado a la Comisión de la IV Asamblea de la Liga de las Naciones.

RAMOS, D. (1924) Cuba en la higiene internacional y el finlaísmo, Habana, Imprenta La Propagandista 